\title{
Novel Approaches to Treating Relapsed and Refractory Multiple Myeloma with a Focus on Recent Approvals of Belantamab Mafodotin and Selinexor
}

\author{
Nisha S Joseph' \\ Yu-Tzu Tai iD ${ }^{2}$ \\ Kenneth C Anderson ${ }^{2}$ \\ Sagar Lonial' \\ 'Winship Cancer Institute, Emory \\ University School of Medicine, Atlanta, \\ GA, USA; '2Dana-Farber Cancer Institute, \\ Harvard University, Boston, MA, USA
}

Correspondence: Kenneth C Anderson Dana-Farber Cancer Institute, Blood Compont, D289, 44 Binney St, Boston, MA, 02115, USA

Tel + I 617 632-2569

Email kenneth_anderson@dfci.harvard.

edu

\begin{abstract}
Though survival outcomes in multiple myeloma patients have improved drastically over the past few decades, there still remains an ongoing need for effective and tolerable treatment options in the relapsed and refractory space. Encouragingly, there have been three recent FDA approvals for triple-class refractory multiple myeloma, and there is promising ongoing development of additional agents with varying novel mechanisms of action. Here, we will review the most recent data on both belantamab mafodotin, an antibody drug conjugate (ADC) targeting BCMA, and selinexor, a first-in-class selective inhibitor of XPO1, as well as touch on some of the recently published data for other immunotherapies in development, namely bispecific T cell engagers, ADCs, and CAR-T cell therapies.
\end{abstract}

Keywords: belantamab mafodotin, belamaf, selinexor, relapsed/refractory myeloma, myeloma

\section{Introduction}

Multiple myeloma is the second most common hematologic malignancy accounting for over 32,000 new cases in the US, and nearly 13,000 deaths in the year $2020 .^{1,2}$ Though incurable, long-term outcomes have improved markedly with median survival now surpassing 10 years for some subsets of patients. ${ }^{3}$ However, effective and tolerable treatment options for relapsed and refractory myeloma (RRMM) patients remains an ongoing unmet need with a median overall survival (OS) of 9.2 months in triple refractory patients, and 5.6 months in penta-refractory patients. ${ }^{4}$ There have been two exciting recent approvals for both selinexor and belantamab mafodotin for triple-class refractory myeloma patients. With more and more treatment options in this space, the challenge now lies in the sequencing and combinations of these therapies, recognizing the importance of utilizing agents with differing mechanisms of action to enhance synergy and optimize outcomes. Here, we provide an overview of the recent developments in RRMM highlighting the recently approved antibody-drug conjugate (ADC), belantamab mafodotin, as well as discussing selinexor and other anti-BCMA and novel-targeted immunotherapies under ongoing investigation in RRMM including other ADCs, bispecific $\mathrm{T}$ cell engagers (BITEs), and CAR-T cell therapy. 


\section{Belantamab Mafodotin}

Belantamab mafodotin, or belantamab, is a first-in-class antibody-drug conjugate targeting B-cell maturation antigen, or BCMA, a transmembrane glycoprotein and member of the tumor necrosis factor (TNF) receptor superfamily that plays an essential role in the development and survival of plasma cells. BCMA is highly expressed on myeloma cells thus making it an ideal target in myeloma treatment. ${ }^{5}$ Belantamab is a humanized monoclonal afucosylated antiBCMA antibody conjugated to the payload MMAF, or monomethyl auristatin F, a microtubule inhibitor that promotes immunogenic cell death. Preclinical models have demonstrated myeloma cell death via both immuneindependent and immune-dependent mechanisms of action including antibody-dependent cellular cytotoxicity (ADCC), antibody-dependent cellular phagocytosis (ADCP), and immunogenic cell death (ICD). ${ }^{6,7}$ Belantamab was approved in August 2020 for the treatment of heavily pretreated RRMM patients with four prior therapies including an immunomodulatory drug (IMID), a proteasome inhibitor (PI), and an anti-CD38 antibody. The FDA-approved dose is $2.5 \mathrm{mg} / \mathrm{kg}$ administered as an intravenous infusion every three weeks. (BLENREP prescribing information)

\section{DREAMM-2}

The approval of belantamab followed the pivotal randomized open-label Phase 2 DREAMM-2 study which demonstrated an initial overall response rate (ORR) of $31 \%$ at the $2.5 \mathrm{mg} / \mathrm{kg}$ dosing in heavily pretreated patients. ${ }^{8,9}$ Nearly 200 patients at over 58 myeloma centers in eight countries were enrolled, meeting eligibility of three or more prior lines of therapy, disease refractory to both IMIDs and PIs, and either refractory and/or intolerant to an anti-CD38 antibody. ${ }^{8}$ At 13 months of follow-up, the ORR was $32 \%$ with an estimated median duration of response (DOR) of 11 months and estimated median overall survival (mOS) of 13.7 months. ${ }^{8,10}$ Moreover, subset analysis of patients with 3-6 prior lines compared to patients that had received $>7$ prior lines showed similar responses rates of $34 \%$ vs $30 \%$, and $\geq$ VGPR $17 \%$ vs $20 \%$, respectively. There was also no difference in $\operatorname{mOS}(13.7$ vs 13.4 months) or median DOR (11 vs 13 months), illustrating that the safety and efficacy of single-agent belantamab is not significantly impacted by the number of prior lines of therapy and remains a useful treatment option even in heavily pretreated patients with otherwise limited treatment options. ${ }^{11}$ Evaluation of response rates stratified by presence of high-risk cytogenetics, including $\mathrm{t}$ $(4 ; 14), \mathrm{t}(14 ; 16)$, del17p or $+1 \mathrm{q} 21$, demonstrated consistent response rates compared to the larger population with an ORR of $27 \%$ in the $2.5 \mathrm{mg} / \mathrm{kg}$ group, and $30 \%$ in the $3.4 \mathrm{mg} / \mathrm{kg}$ group. ${ }^{12}$

In terms of safety, infusion-related reactions (IRR) were noted in $21 \%(20 / 95)$ of patients, mostly mild or moderate with no grade 4 reactions, and a majority of the IRRs occurred in cycle 1 . Given the low rate of IRR in DREAMM-1, premedications were not required in the Phase II study, though recommended, particularly in patients who had already experienced an IRR on study. Preventative medications were administered per the treating physician's discretion, and the frequency of analgesics, antihistamines, and steroid administration were $75 \%, 75 \%$, and $50 \%$, respectively. ${ }^{13}$

Ocular toxicity was the more concerning adverse event and a common occurrence in DREAMM-2 including dry eye, blurry vision or change in BCVA (best corrected visual acuity), keratopathy and corneal microcyst-like epithelial changes (MECs). Almost three-quarters of the patients experienced some degree of keratopathy, with $60 \%$ of these patients having grade 2 keratopathy or higher and $48 \%$ of these patients having more than one event. Fifty-six percent of patients experienced symptoms such as blurry vision, dry eye or $\geq 2$ line decline in BCVA. Seventeen patients (18\%) experienced a decline in BCVA to $20 / 50$ or worse, and three patients $(3 \%)$ discontinued therapy due to corneal adverse events. In terms of recovery from these ocular events, post hoc analysis at 13 months follow-up showed that $77 \%$ of these patients had resolution of symptoms while on therapy, and another $20 \%$ experienced recovery following completion of therapy. At last follow-up, $84 \%$ of patients with grade 3/4 keratopathy were either improving or recovered, and importantly, no patient had permanent vision loss. ${ }^{14}$ Though ocular symptoms are concerning, review of patient-reported outcomes (PRO) revealed that responders reported high levels of satisfaction while on therapy despite these issues, suggesting that these ocular toxicities are manageable particularly when effective. ${ }^{15}$ Furthermore, data demonstrate that though ocular toxicity is a frequent and expected adverse event seen with belantamab, these events can be managed appropriately with dose delays and modifications allowing for a majority of patients to recover.

Given this expected and common need for dose interruptions and delays, a relevant question is whether or not response is maintained despite these disruptions in 
treatment. In DREAMM-2, 41\% (39/95) of patients in the $2.5 \mathrm{mg} / \mathrm{kg}$ arm and $48 \%$ (48/99) of patients in the $3.4 \mathrm{mg} /$ $\mathrm{kg}$ arm experienced a dose delay. The most common reason for delay was keratopathy, and the average duration of dose delay was 42 days (range, 4-212) and 23 days (4149 ) in the $2.5 \mathrm{mg} / \mathrm{kg}$ and $3.4 \mathrm{mg} / \mathrm{kg}$ arms, respectively. Of those patients experiencing a prolonged delay ( $>63$ days), $\sim 85 \%$ maintained a clinical benefit. Only two patients in the $2.5 \mathrm{mg} / \mathrm{kg}$ arm and three patients in the $3.4 \mathrm{mg} / \mathrm{kg}$ arm met the criteria for disease progression. In fact, in the $2.5 \mathrm{mg} / \mathrm{kg}$ and $3.4 \mathrm{mg} / \mathrm{kg}$ arm respectively, $38 \%$ and $32 \%$ of patients deepened their clinical response while $38 \%$ and $42 \%$ maintained the same response at time of dose delay. Moreover, $80 \%$ of patients with grade $3 / 4$ keratopathy had improvement in ocular findings to grade 2 or less again confirming the practical need and utility of dose delays. $^{16}$

Based on the promising results of DREAMM-2 and given its multimodal mechanism of action, belantamab was an ideal candidate for use in RRMM combination regimens. To date, belantamab is currently being studied in combination with pomalidomide, bortezomib, lenalidomide, PD-1 inhibitors and several novel small molecules.

\section{DREAMM-3}

DREAMM-3 is a randomized, open-label multicenter Phase 3 trial investigating the safety and efficacy of belantamab in combination with pomalidomide and dexamethasone (bela/pom/dex) in patients with RRMM having progressed on at least two prior lines of therapy. ${ }^{17}$ Belantamab is given at either $1.92 \mathrm{mg} / \mathrm{kg}, 2.5 \mathrm{mg} / \mathrm{kg}$ single dose, $2.5 \mathrm{mg} / \mathrm{kg}$ as a split dose on days 1 and 8 , or $3.4 \mathrm{mg} /$ $\mathrm{kg}$ as a split dose on days 1 and 8 . Pomalidomide is dosed at $4 \mathrm{mg}$ on days 1-21 of a 28-day cycle, and dexamethasone is dosed at either $20 \mathrm{mg}$ or $40 \mathrm{mg}$ based on age on days $1,8,15$, and 22 . This study is ongoing and results are eagerly awaited.

Updated results of the ALGONQUINN study, a twopart phase 1 multicenter study conducted by the Canadian Myeloma Research Group, were presented at the most recent ASH Annual Meeting on a cohort of 20 patients who had received belantamab at $2.5 \mathrm{mg} / \mathrm{kg}$ either as a single or split dose also with pom/dex. Belantamab was dosed at either 1.92 or $2.5 \mathrm{mg} / \mathrm{kg}$ given every four weeks, given as a loading dose of $2.5 \mathrm{mg} / \mathrm{kg}$ on C1D1 and subsequently dose reduced to $1.92 \mathrm{mg} / \mathrm{kg}$ for cycle 2 onwards, or dosed at 2.5 or $3.4 \mathrm{mg} / \mathrm{kg}$ split equally on divided dose on days 1 and 8 of each cycle. Pomalidomide and dexamethasone were dosed standardly at $4 \mathrm{mg}$ on days 1-21 and 40 weekly on a 28-day cycle, respectively. The maximum tolerated dose (MTD) of bela/pom/dex was established at $2.5 \mathrm{mg} / \mathrm{kg}$ dosed as either a single or split dose on days 1 and 8 every 4 weeks. Initial results from the combined cohort of 20 patients receiving belantamab at the initial dose $2.5 \mathrm{mg} / \mathrm{kg}$ were presented. Patients were heavily pretreated with a median of three prior lines of therapy, $89.2 \%$ of patients were lenalidomide refractory, $73 \%$ were refractory to both lenalidomide and a proteasome inhibitor, and roughly a third of patients were triple class refractory. At the time of analysis, for the entire cohort of 37 patients, $76 \%$ of patients continued on study with median of nine cycles of therapy. Regarding toxicity, $43 \%$ of patients experienced a serious adverse event (SAE), but only $24.3 \%$ of those were considered treatment-related. Thirty-eight percent of patients experienced an adverse event that led to dose reduction, and three-quarters of patients required dose interruption. The most frequent grade 3 or higher adverse events included keratopathy $(54.1 \%)$, neutropenia $(40.5 \%)$, thrombocytopenia $(32.4 \%)$, decreased visual acuity $(16.2 \%)$, and fatigue $(10.8 \%)$. Keratopathy of all grades occurred in $75 \%$ of patients, but only $16.2 \%$ experienced grade 3 or higher decrease in visual acuity. In patients receiving the $1.92 \mathrm{mg} /$ $\mathrm{kg}$ dose of belantamab, the rate of all grade keratopathy was significantly lower at $25 \%$, however, there was not a significant difference in the rate of meaningful change in visual acuity $-16.2 \%$ in the $2.5 \mathrm{mg} / \mathrm{kg}$ group compared to $17 \%$ in the $1.92 \mathrm{mg} / \mathrm{kg}$ group. At a median follow-up of 7.8 months, the ORR for the 34 evaluable patients was $88 \%$ with a $\geq$ VGPR of $68 \%$. This depth of response was maintained in IMID/PI refractory patients with ORR 92\% and $\geq$ VGPR of $75 \%$, and in triple class refractory patients with an ORR of $100 \%$ and $\geq$ VGPR of $75 \%$. Notably the $\mathrm{mPFS}$ was not reached in the IMID/PI refractory group, and was 11.1 months in the triple class refractory subset. Subgroup analysis of only the $2.5 \mathrm{mg} / \mathrm{kg}$ patients showed an extremely impressive ORR of $100 \%$, and notably, $a \geq V G P R$ of $100 \%$. In comparison with other triplet regimens containing pom/dex, bela/pom/dex compares very favorably. The concern remains the toxicity, particularly ocular, prompting the further investigation of alternative dosing schedules such as belantamab dosed at every eightor even 12-week intervals, in attempts to improve the safety profile. This strategy is further supported by sustained responses in patients despite multiple dose 
interruptions as described earlier in the DREAMM-2 post hoc analysis. ${ }^{18}$

As a follow-up, DREAMM-8, is an ongoing phase 3 study comparing bela/pom/dex versus pomalidomide, bortezomib and dexamethasone (pom/bort/dex) in RRMM. It is a two-arm, multicenter, randomized study that began in October 2020 with intent of enrolling 450 patients worldwide. ${ }^{19}$ Pom/bort/dex is an approved triplet regimen for RRMM based on the OPTIMISMM trial showing or ORR $82 \%$ and mPFS of 11.2 months. ${ }^{20}$ Belantamab will be dosed at $2.5 \mathrm{mg} / \mathrm{kg}$ on C1D1 and subsequently reduced to the $1.92 \mathrm{mg} / \mathrm{kg}$ dose with cycle 2 onwards. ${ }^{19}$

\section{DREAMM-4}

DREAMM-4 is an ongoing single-arm, open-label, twopart Phase I/II study designed to investigate belantamab in combination with pembrolizumab in attempts to induce and enhance belantamab antimyeloma activity. In the part 1 dose escalation phase, belantamab at either 2.5 or $3.4 \mathrm{mg} / \mathrm{kg}$ is administered in combination with flat dose pembrolizumab $200 \mathrm{mg}$ every three weeks. Interim analysis included 13 patients with a median of five prior lines, and $31 \%$ of patients had high-risk cytogenetics. At a median follow-up of almost seven months for the belantamab $2.5 \mathrm{mg} / \mathrm{kg}$ cohort and four months for the $3.4 \mathrm{mg} / \mathrm{kg}$ cohort, the median number of cycles administered were six and three, respectively. Five of the patients in the $2.5 \mathrm{mg} /$ $\mathrm{kg}$ group and four in the $3.4 \mathrm{mg} / \mathrm{kg}$ group required dose interruptions. Unsurprisingly, keratopathy was the most common treatment-related $\mathrm{AE}$ in both groups, though there was a higher rate of grade 3-4 keratopathy in the $2.5 \mathrm{mg} / \mathrm{kg}$ group (33\% vs $0 \%$ ) leading to a higher rate of dose delays and modifications in the $2.5 \mathrm{mg} / \mathrm{kg}$ group compared to the $3.4 \mathrm{mg} / \mathrm{kg}$ group (67\% and $33 \%$ vs $14 \%$ and $0 \%$, respectively). The ORR was $67 \%$ in the $2.5 \mathrm{mg} /$ $\mathrm{kg}$ group with $\geq \mathrm{VGPR}$ of $33 \%$, and $43 \%$ in the $3.4 \mathrm{mg} / \mathrm{kg}$ group. Two patients, both in the $3.4 \mathrm{mg} / \mathrm{kg}$ group, have experienced progressive disease thus far. The safety profile has been acceptable with no dose limiting toxicities (DLTs) or fatal events to date, and consistent with prior belantamab studies. The part 2 expansion phase is currently recruiting. ${ }^{21}$

\section{DREAMM-5}

DREAMM-5 is an ongoing platform study investigating combination strategies with belantamab and several novel small molecule agents. The trial contains four sub-studies and is actively recruiting. Sub-study 1 explores the combination of belantamab with GSK3174998, an antiOX40 antibody based on preclinical data supporting the combination. Sub-study 2 combines belantamab with feladilimab, an anti-ICOS IgG4 antibody. ICOS is a costimulatory receptor and member of the CD28 family that plays a key role in the survival and function of $\mathrm{T}$ cells. Feladilimab was designed to enhance $\mathrm{T}$ cell responses and promote myeloma cell death without depleting ICOS-expressing cells. Sub-study 3 explores the addition of nirogacestat, a gamma secretase inhibitor which prevents the cleavage of transmembrane BCMA. Lastly, sub-study 4 is exploring the combination of dostarlimab, an anti-PD1 IgG4 monoclonal antibody with belantamab. ${ }^{22}$ Enrollment is ongoing and results of these sub-studies are eagerly awaited.

\section{DREAMM-6}

DREAMM-6 is an ongoing two-arm, two-part Phase I/II trial investigating the safety and efficacy of belantamab in combination with lenalidomide and dexamethasone (len/ dex), and bortezomib and dexamethasone (bort/dex) in patients treated with at least one prior line of therapy. In the dose expansion phase of the study, there are four separate cohorts examining each combination regimen at either belantamab $2.5 \mathrm{mg} / \mathrm{kg}$ or $3.4 \mathrm{mg} / \mathrm{kg}$ every three weeks. ${ }^{23}$ Eighteen patients have been enrolled on the $2.5 \mathrm{mg} / \mathrm{kg}$ arm, and notably, bortezomib refractory patients were not excluded from the study with $89 \%$ of patients having had prior bortezomib exposure, and 50\% having had prior daratumumab exposure. One quarter of the patients had high-risk cytogenetics and one quarter of the patients had extramedullary disease. The ORR was $78 \%$ for the entire cohort, $75 \%$ (95\%CI: $47.6-92.7)$ for patients with prior bortezomib exposure, and 67\% (95\% CI: 29.9-92.5) for those patients with prior daratumumab exposure. VGPR or better rates were similarly impressive at $67 \%$ for the entire cohort, and $63 \%$ and $44 \%$ for the bortezomib exposed and daratumumab exposed, respectively. Ocular toxicity, primarily keratopathy, was a common occurrence, but only three patients experienced grade 3 keratopathy, and all cases were manageable with appropriate dose delays and reductions. Thrombocytopenia was also a common adverse event in $78 \%$ of patients, and two-thirds of patients experienced grade 3 or 4 thrombocytopenia. To date, the bela/bort/dex combination has shown an acceptable safety profile in RRMM and data supports phase 3 studies for this combination in early relapse. ${ }^{24}$ 
There are several ongoing DREAMM studies investigating alternative dosing scheduled and/or combination regimens with belantamab. DREAMM-7 is an ongoing randomized, open-label, multicenter Phase III study assessing the safety and efficacy of belantamab with bort/dex vs daratumumab with bort/dex in patients with RRMM, based off of the promising results of the phase I/II DREAMM-6 study. As of November 2020, 108 patients had been enrolled in 15 participating countries worldwide. ${ }^{25}$ DREAMM-8, as discussed above, is an ongoing phase III trial comparing bela/pom/dex with pom/bort/dex. DREAMM-9 is planned and set to examine the efficacy of belantamab in combination with lenalidomide, bortezomib and dexamethasone (RVD) vs RVD alone in transplant-ineligible newly diagnosed myeloma patients (NDMM). We eagerly await the results of ongoing and upcoming studies to provide further insight and clarification both on the safety and efficacy of belantamab in combination regimens as well as earlier incorporation of this agent in the disease course.

It is also worth noting, given the number of novel antiBCMA therapies in development, it is important to understand if treatment with one would affect the efficacy of another agent given either sequentially or later on in the treatment course. To date, there are no prospective clinical trial data to elucidate this question. What has been shown, though, is that despite downregulation of BCMA following BCMA-directed therapy such as CAR-T, this expression is maintained at time of disease progression suggesting response to other anti-BCMA agents is possible. ${ }^{26}$ Cohen et al have also published case reports describing responses to anti-BCMA therapy following CAR-T and suggest though the target is the same, differing mechanisms of action allow for response. ${ }^{27}$ This will be an important area of study and focus as we incorporate these therapies more and more and earlier into treatment courses.

In summary, belantamab mafodotin is a promising novel therapeutic in the relapsed and refractory space offering options for patients now who have progressed through multiple lines of therapy. Ocular toxicity, though manageable and often resolving with time and appropriate dose delays and reductions, remains a concern. Clinical investigation into use of belantamab in earlier relapse and newly diagnosed myeloma as well as in novel combination strategies is extremely active. Looking forward, our understanding will only deepen on how to best utilize belantamab to better outcomes in RRMM patients.

\section{Selinexor}

In addition to belantamab, selinexor has also become a viable treatment option for multiple relapsed myeloma patients. Selinexor is a first-in-class, oral, selective inhibitor of the nuclear export protein exportin 1 (XPO1), which is overexpressed in myeloma cells and associated with poor prognosis and resistance to therapy. Selinexor irreversibly binds to Cys528 in the cargo-binding pocket of XPO1 leading to the nuclear accumulation and activation of key tumor suppressor proteins, and subsequently leading to cell cycle arrest and apoptosis. ${ }^{28-31}$ Selinexor was granted accelerated approval in 2019 in combination with dexamethasone (sel/dex) based on results from the phase IIb STORM trial for RRMM patients who have received at least four prior therapies and are penta-refractory (refractory to at least two PIs, two IMIDS and anti-CD38 monoclonal antibody). Sel/dex was found to have an ORR of $26 \%$ with a median PFS of 3.7 months and a mOS of 8.6 months in this very heavily pretreated population. The most common adverse events were primarily thrombocytopenia (58\%), anemia (44\%), and fatigue (25\%). Nausea and anorexia were also frequent, occurring in $72 \%$ and $56 \%$ of patients, respectively. ${ }^{32}$

Though there is a critical need for novel therapies in this treatment space, the initial FDA approved dose and schedule of selinexor proved difficult in the clinical setting with fairly significant gastrointestinal toxicity including nausea, anorexia and weight loss. What has proven more promising is identifying synergistic partners in combination with selinexor that allow for decreased doses of selinexor and thus a more tolerable treatment regimen. It is worth noting though that in a post hoc analysis using matching-adjusted indirect comparisons (MAIC) to compare cross-study results of DREAMM-2 to STORM (sel/ dex) and MAMMOTH, a retrospective analysis of RRMM patients following standard of care, belantamab monotherapy was found to have superior efficacy in terms of improved overall survival compared to sel/dex (HR: 0.60 ; 95\%CI: $0.39-0.94 ; p=0.025$ ) and SOC (HR: 0.33; 95\%CI: $0.17-0.63 ; p<0.001)$. In addition, improved duration of response was seen with belantamab, though no statistically significant difference was seen in terms of progression free survival, time to response or overall response rates. ${ }^{33}$ Conversely, with the recent approval of Ide-cel and increased use of BCMA-targeting agents, selinexor is a potentially useful agent in the context of relapse following anti-BCMA treatment. Identification of seven patients treated with selinexor immediately following 
progression after CAR-T cell therapy across multiple studies, all heavily pretreated and with high-risk cytogenetics, found that $6 / 7$ patients achieved at least a PR. One patient achieved a sCR and three patients achieved VGPR. Notably, of the patients who achieved at least a PR, four of these responses occurred within the first cycle of treatment. $^{34}$

Just as with belantamab, selinexor combination regimens have been more promising in terms of efficacy and safety profile, and further and more mature data on both of these agents is needed. Preclinical data has shown increased efficacy of selinexor when combined with proteasome inhibitors and steroids due to decreased signaling via the NF- $\kappa$ B pathway. ${ }^{35,36}$ Selinexor with bort/dex was studied in the Phase II randomized, open-label BOSTON trial in RRMM patients having received 1-3 prior lines of therapy. Preclinical data has provided rationale for combining proteasome inhibitors with selinexor, demonstrating synergy through suppression of the NF-kB pathway and increased myeloma cell death in both patient-derived myeloma cells and murine models. ${ }^{35,37}$ In contrast to the STORM trial, selinexor was only given once weekly in combination with weekly bortezomib and low-dose dexamethasone. Efficacy favored the triplet with a median PFS of 13.9 months vs 9.5 months, though increased grade 3-4 adverse events were seen on the triplet arm with higher rates of thrombocytopenia, fatigue, and anemia with the notable exception of decreased rates of peripheral neuropathy as compared to the bort/dex arm. ${ }^{38}$ The results of the BOSTON study led to FDA approval of this combination in December 2020 in RRMM patients who had received at least one prior line of therapy.

The STOMP trial is a multicenter, open-label, randomized phase $1 / 2 b$ study investigating selinexor in dose escalation and expansion phases in multiple combination regimens. One arm investigated selinexor $80 \mathrm{mg}$ weekly, in combination with carfilzomib $56 \mathrm{mg} / \mathrm{m}^{2}$ weekly, and dexamethasone $40 \mathrm{mg}$ weekly, a regimen previous demonstrated to have promising clinical activity in the phase 1 study. ${ }^{39}$ The ORR was $72 \%$ with $\geq$ VGPR of $55 \%$. Thrombocytopenia, nausea, anorexia and fatigue were again common adverse events, but notably, there were no grade 3 or 4 AEs. Again, in combination with other potent agents allowing for reduced doses of selinexor, efficacy is seen with a more manageable safety profile. ${ }^{39-41}$

Another regimen investigated in the STOMP trial is selinexor in combination with pom/dex (SPd). The R2PD was defined as selinexor $80 \mathrm{mg}$ once weekly with pomalidomide $4 \mathrm{mg}$ on days 1-21 and dex $40 \mathrm{mg}$ once weekly on a 28-day cycle. ${ }^{42}$ As of November 2020, 65 patients have been enrolled and 20 patients have been treated at the R2PD with a median of three prior lines of therapy. All patients were exposed to lenalidomide and $75 \%$ were refractory to lenalidomide. At the R2PD, the ORR $60 \%$ with $\geq V G P R$ of $30 \%$. In pomalidomide refractory patients, the ORR was $36 \%$ with $\geq V$ VPR or better of 7.1\%. Median PFS for the entire cohort was 12.2 months, and NR for patients dosed at R2PD. Median DOR was 11.3 months for the entire cohort, and again, NR for R2PD cohort. $^{42}$ Selinexor, daratumumab, and dexamethasone has shown similar depth of response with ORR $73 \%$ and mPFS 12.5 months in anti-CD38 monoclonal antibody naïve patients. ${ }^{43}$ Selinexor, lenalidomide and dexamethasone results from STOMP have been reported for the first 24 patients finding an impressive ORR of $92 \%$ in lennaïve, however, somewhat disappointing ORR of $13 \%$ in len-exposed patients suggesting perhaps increased benefit of this combination earlier in the treatment course. ${ }^{44}$

In addition to belamantamab mafodotin and selinexor, a review of novel therapeutics in RRMM would be incomplete without brief mention of other ADCs, BiTEs, and briefly CAR-T cell therapies under investigation.

\section{Antibody-Drug Conjugates (ADCs)}

As illustrated by the discussion above regarding belantamab, antibody-drug conjugates have significant potential in the treatment of myeloma, and are exciting options as they are "off-the-shelf" therapies and thus readily available to a broad range of patients. Belantamab mafodotin is the most clinically studied to date, but others are in development in early phase trials also targeting BCMA as well as alternative targets including CD38, CD74, and CD46. ${ }^{45}$

\section{MEDI2228}

MEDI2228 is an antibody-drug conjugate comprised of a fully human BCMA antibody conjugated via a proteasecleavable linker to pyrrolobenzodiazepine (PBD), a DNA cross-linking agent. The Phase I, first-in-human doseescalation study at the maximum tolerated dose (MTD) of $0.14 \mathrm{mg} / \mathrm{kg}$ administered intravenously every three weeks found an ORR of $65.9 \%$ with a $\geq$ VGPR of $24.4 \%$. The most common grade 3-4 adverse events were expected class effects, however unexpectedly, $60 \%$ of patients experienced photophobia. These events were predominantly of grade 1 and 2 , but $17 \%$ of these patients experienced grade 3-4 photophobia. The median time to 
onset of these ocular symptoms was two to three cycles with $37 \%$ of patients experiencing an improvement in symptoms over time. Overall, MEDI2228 has shown clinical efficacy and a reasonable safety profile in a heavily pretreated RRMM population, however further exploration will delve into alternative dosing schedules with the aim of mitigating ocular toxicity. ${ }^{46}$

\section{Bispecific T Cell Engagers}

Bispecific $\mathrm{T}$ cell engagers or BITEs have also become an exciting treatment option in RRMM patients, both targeting BCMA as well as other novel targets.

\section{Teclistamab}

Teclistamab (JNJ-64007957) is a humanized BCMA-CD3 bispecific IgG4 antibody designed to redirect CD3+ T-cells to BCMA-expressing myeloma cells. Preliminary results on intravenous dosing showed an ORR of $67 \%$ dosed at $270 \mu \mathrm{g} / \mathrm{kg}$. More recently, data on subcutaneous dosing has been promising with an ORR of $73 \%$ and $\geq$ VGPR of $55 \%$ at the R2PD of $1500 \mu \mathrm{g} / \mathrm{kg}$ once weekly. There was no grade 3-4 CRS or neurotoxicity of any grade with the subcutaneous dosing. Thirty-seven percent of patients experienced grade 1-2 CRS though there were no resultant treatment discontinuations. Injection site reactions were common but all grade 1-2. Besides CRS, the most common adverse events were hematologic, primarily neutropenia. ${ }^{47}$ In comparison, though modest, there were higher rates of neurotoxicity with intravenous dosing. ${ }^{48} \mathrm{To}$ date, teclistimab has shown encouraging efficacy and safety profile and further data from the ongoing Phase 1 , the recently enrolling Phase 2 expansion monotherapy study as well as future combination studies are awaited. Such combinations under active investigation include teclistimab with nirogacestat (NCT04722146), as well as teclistimab with daratumumab with or without pomalidomide and talquetamab, discussed below (NCT04108195).

\section{REGN5458}

REGN5458 is another $\mathrm{BCMA} \times \mathrm{CD} 3 \mathrm{BiTE}$ that has shown promising preliminary efficacy and safety. The first-inhuman Phase I trial is ongoing, administered REGN5458 as a split dose for the first two weeks and then weekly from weeks $3-16$, followed by maintenance dosing every two weeks until disease progression. Nonhematologic grade 3-4 adverse events were uncommon, and $18 \%$ of patients experienced grade 3-4 infections with a majority of these being grade 3 pneumonia.
CRS was all grade 1-2 and occurred in $38 \%$ of patients. At a median follow up of 2.6 months, the ORR of all dose levels was $39 \%$ with $95 \%$ of responders achieving $\geq$ VGPR. MRD testing was done on seven evaluable patients and four were MRD negative at $10^{-5}$. At the highest dose level currently reported (DL6), the ORR was $62.5 \%$. Responses were seen early and deepened with time with a median duration of response of six months. ${ }^{49}$ The phase 2 portion of the study is currently enrolling.

\section{CC-93269}

CC-93269 is a two-arm humanized bi-specific T cell engager that binds bivalently to BCMA and monovalently to CD3 inducing $\mathrm{T}$ cell activation and subsequent T cells mediate myeloma cell death. ${ }^{50}$ Though the R2PD has not yet been reached, doses have ranged from $0.5 \mathrm{mg}$ up to $10 \mathrm{mg}$ with a median of four cycles. Of the 12 patients treated with doses either at or above $6 \mathrm{mg}$, the ORR was $83 \%$ with $\geq$ VGPR of $58 \%$ and $33 \%$ achieved a sCR. Among patients receiving $10 \mathrm{mg}$, the sCR rate was $44 \%$. Impressively, $75 \%$ of these patients have achieved MRD negativity. Nearly all patients (97\%) experienced TEAEs, predominantly cytopenias or infectious, and $73 \%$ of these were grade 3 or higher. Ninety percent of patients experienced CRS, though a majority of these were grade 1/ 2. Though the rate of CRS was high with CC-93269, the impressive responses including MRD-negative sCRs in such a heavily pretreated population warrant further investigation. ${ }^{51}$

\section{TNB-383b}

TNB-383B is a fully human triple-chain $B C M A \times C D 3$ BiTE, though the compound was specifically designed in attempts to reduce toxicity without sacrificing efficacy. TNB-383B contains a unique anti-CD3 moiety to reduce the risk of CRS, two BCMA domains favoring cell surface BCMA binding, and a silenced IgG4 backbone to circumvent nonspecific $\mathrm{T}$ cell activation and provide a longer half-life. Preliminary results from the first in human phase I study showed an impressive ORR of $80 \%$ with $\geq$ VGPR $73 \%$, and $\geq \mathrm{CR} 13 \%$ at doses above $40 \mathrm{mg}$. The study is ongoing and R2PD has not yet been reached, but 22 of the 27 patients have ongoing response with a median of 4.5 months on study. The most common nonhematologic AE was CRS (45\%), seen at higher rates at the $<40 \mathrm{mg}$ doses, but there was no grade 3 or higher CRS and only one episode of recurrence outside of cycle $1 .^{52}$ 
Thus far, TNB-383B has shown a manageable safety profile and good efficacy, but what also allows it to stand out is the convenient every three weeks dosing schedule.

\section{PF-3 I 35}

PF-06863135 (PF-3135) is a humanized bispecific $\mathrm{BCMA} \times \mathrm{CD} 3$ antibody currently being evaluated as subcutaneous dosing at $80,130,215$, and $360 \mu \mathrm{g} / \mathrm{kg}$ weekly. As of April 2020, 18 patients had been enrolled and notably four of these patients had received prior anti-BCMA ADC or CART cell therapy. CRS was reported in 11 (61\%) of the patients, $50 \%$ G1 and $11 \%$ G2. The ORR of the entire cohort was $33 \%$, but notably at the highest two dose levels (215 and 360 $\mu \mathrm{g} / \mathrm{kg}$ ), the ORR was $75 \%$ (6/8 patients) with four patients achieving $\geq$ VGPR. Dose escalation is ongoing and further results at these higher dose levels are awaited.

\section{Bispecific T Cell Engagers: Non-BCMA Targets}

Given the promising efficacy seen with BiTEs, further interest has been generated in other non-BCMA targets in attempts to create diverse therapy options for RRMM patients.

\section{Talquetamab}

Talquetamab is an exciting first-in-class GPRC $5 \mathrm{D} \times \mathrm{CD} 3$ BiTE showing antimyeloma activity in preclinical models. GPRC5D is an orphan G protein coupled receptor of unknown function that is highly expressed on malignant plasma cells. In the large phase 1 dose escalation, first-inhuman trial, 157 patients have been enrolled to date and the R2PD was $405 \mu \mathrm{g} / \mathrm{kg}$ administered subcutaneously. Prior BCMA was allowed, though neither the exact number of these patient nor efficacy in this subset has yet been reported. The ORR was almost $70 \%$ and $\geq$ VGPR $39 \%$. CRS was all grade $1-2$, and there was only one instance of neurotoxicity at the R2PD which resolved. Though cytopenias were common, infections were infrequent and early grade, somewhat surprising in this patient population. ${ }^{53}$ Further data from the ongoing dose expansion and planned phase 2 studies are eagerly awaited, as well as data on combination regimens with subcutaneous daratumumab (NCT04108195).

\section{Cevostamab}

Cevostamab, or BFCR4350A, is a humanized IgG bispecific antibody that binds to both membrane-bound Fc receptor-homolog 5 (FCRH5) on myeloma cells and CD3 $+\mathrm{T}$ cells. FCRH5 is highly expressed on myeloma cells, and thus another attractive therapeutic target. In the ongoing phase 1 dose escalation and expansion study, cevostamab was dosed intravenously every three weeks in RRMM patients. Of note, those with prior treatment with CAR T-cells, BiTEs, and ADCs, including those targeting BCMA (21\%), were eligible. R2PD has not yet been reached, but at doses exceeding $20 \mathrm{mg}$, the ORR is $53 \%$ with $\geq$ VGPR $32 \%$ and $\geq$ CR $18 \%$. At the highest dose levels tested, the ORR exceeds $60 \%$ with $\geq$ VGPR $28 \%$, $\geq \mathrm{CR} 11 \%$ and $\mathrm{sCR}$ rate of $6 \%$. The median time to response was 30 days, and response has been unrelated to FCRH5 expression. ${ }^{54}$ Encouragingly, responses have been seen in patients with prior BCMA therapy and in patients that have discontinued study drug. ${ }^{55}$ Again, though this is preliminary data, the response rates are very encouraging, particularly in a difficult to treat population particularly those with prior BCMA exposure. The dose escalation and expansion phases are ongoing, and certainly combination regimens with cevostamab are likely on the horizon.

\section{CELMoDs}

lberdomide (CC-220)

A discussion of promising emerging therapies in the relapsed space would be incomplete without mention of the novel CeLMOD agents CC-220, or iberdomide, and CC-92480. Iberdomide is a novel and potent cereblon E3 ligase modulator (CELMoD) that has shown increased potency in preclinical models compared to lenalidomide and pomalidomide as seen by increased degradation of the substrates Aiolos and Ikaros and greater rates of apoptosis. ${ }^{56}$ In addition, this activity was also seen in both lenalidomide-refractory and pomalidomiderefractory cell lines, and synergy was demonstrated with daratumumab, bortezomib, and dexamethasone. ${ }^{56}$ The ongoing Phase 1b/2a multicenter, open-label, doseescalation study (NCT02773030) is evaluating iberdomide monotherapy as well in combination with daratumumab and bortezomib in RRMM. In combination with dexamethasone, the R2PD for iberdomide was $1.6 \mathrm{mg}$ and the reported ORR was $31 \%$ with a majority of grade 3-4 AEs being cytopenias, fatigue and neuropathy. In combination with dara/dex, the ORR was $42 \%$ with $\geq$ VGPR $19.2 \%$. When selecting out those refractory to daratumumab, ORR was $60 \%$ in dara- naïve/exposed patients compared to $30 \%$ 
in dara-refractory patients. In combination with bort/dex, the ORR was $61 \%$ with $\geq$ VGPR $26 \%$ with responses seen even in bortezomib-refractory patients. In both cohorts, AEs were mostly hematologic and the most common nonhematologic AE was infection. There is ongoing investigation into combination regimens and into moving this treatment option into the newly diagnosed/early relapse setting.

\section{CC-92480}

CC-92480 is another novel CELMoD currently under investigation in an ongoing open-label, multicenter Phase 1 study in combination with dexamethasone in patients with RRMM. Initial results are promising with an ORR $54.5 \%$ at the R2PD of $1.0 \mathrm{mg}$ on days $1-21$ every 28 days. Notably, this response was maintained even in patients with pomalidomide-refractory disease. ${ }^{57}$

\section{CAR-T Cell Therapy}

Though a full discussion of CAR-T cell therapies in RRMM is outside of the scope of this review, given the exciting developments and potential of this approach, it is worth highlighting a few of the notable constructs.

In the initial phase I CRB-401 study, Idecabtagene vicleucel or Ide-cel, achieved high response rates with an ORR $85 \%$ and $\mathrm{CR}$ of $45 \%$, however, duration of response was limited with a median PFS of 11.8 months. ${ }^{58}$ The subsequent Phase 2 KarMMa trial confirmed the efficacy of Ide-cel dosed at $150-450 \times 10^{6}$ CAR-T cells with an ORR $73 \%$ and CR rate of $33 \%$, with one-third of these patients achieving an MRD negative CR. ${ }^{59}$ Updated results from CRB-402, investigating the same CAR-T design as ideleucel, but coculturing the produce with a phosphoinositide 3-kinase inhibitor (PI3K) to enrich the product for memory-like T cells, reported an ORR of $84 \%$ at $450 \times 10^{6}$ dose with a median duration of response of 17 months across all doses in 69 patients. ${ }^{60}$ KarMMA-3, a Phase 3 study comparing Ide-cel to standard of care in RRM, and KarMMa-4, a phase 1 study investigating Ide-cel in high-risk NDMM, are ongoing. ${ }^{61}$ Ide-cel was FDA approved in March 2021 for treatment of RRMM with four or more prior lines of therapy.

Ciltacabtagene autoleucel, or Cilta-cel, previously known as JNJ-68284528 and LCAR-B38M, is a second generation CAR-T with 2 BCMA binding domains evaluated in the Phase 1b/2 CARTITUDE-1 study. In nearly 100 patients, the ORR was $97 \%$ with $\geq$ VGPR $93 \%$ and $\mathrm{sCR}$ rate of $67 \%$. The mPFS was not reached, but the 12 months PFS was $76.6 \%$ and 12 month OS was $89 \%$. Over $90 \%$ of patients achieved MRD negativity and $72 \%$ of patients remain on study. CRS was predominantly grade $1-2$, though seen in $95 \%$ of patients with delayed median onset of seven days (range, 1-12) perhaps due to slightly delayed CAR-T expansion seen with Cilta-cel compared to other products. ${ }^{62}$ Further investigation into Cilta-cel in different treatment lines and combinations are ongoing in CARTITUDE-2 (NCT04133636) and CARTITUDE-4 (NCT04181827). Orva-cel, also known as orvacabtagene autoleucel or JCARH125, is another BCMA-directed CART cell product evaluated in the Phase I EVOLVE study, and showed impressive efficacy with an ORR 91\%, $\geq$ CR 39\% at all dose levels. ${ }^{63}$

CAR-T cell therapy in myeloma is an exciting and promising therapeutic strategy with clear efficacy, but ongoing challenges with durability of response. Further investigation is ongoing to optimize the product include co-administration with drugs such as gamma secretase inhibitors that upregulate BCMA expression, ${ }^{64}$ using "armored" cars or immune modulators such as lenalidomide to downregulate the host response and increase CAR-T persistence, ${ }^{65}$ as well as ongoing investigation into "off the shelf" allogeneic CAR-T products which have shown promising results to date. ${ }^{63}$

\section{Conclusion}

The field of myeloma has seen such significant progress in the past 10 to 15 years, with more and more patients living longer as though they have a chronic disease. Recent approval of the anti-BCMA antibody-drug conjugate, belantamab mafodotin, as well as selinexor, has provided new therapeutic options for RRMM where previously there were none. The identification of BCMA as a new target in antimyeloma therapies has led to further and ongoing development of immunotherapy in multiple myeloma including antibody-drug conjugates, bispecific $\mathrm{T}$ cell engagers, and CAR-T cell therapy. As the treatment landscape in myeloma continues to evolve, ongoing and future studies will continue to investigate these therapies in combination and earlier in disease course. The hope is that in the near future we will be able to achieve long-term disease free survival by obtaining deep responses with a combination of immune and targeted agents, and then learn how to sustain these responses by restoring an immune response in patients against their own disease.

\section{Disclosure}

Dr Kenneth C Anderson reports personal fees from Pfizer, Amgen, AstraZeneca, Janssen, Precision Biosciences, 
Mana, Windmill, Raqia, C4 Therapeutics, and oncopep, during the conduct of the study. Dr Sagar Lonial reports grants and/or personal fees from Takeda, Janssen, BMS, Celgene, GSK, AbbVie, Bluebird, Novartis, and Pfizer; personal fees from and board of directors for $\mathrm{TG}$ Therapeutics, during the conduct of the study. The authors report no other conflicts of interest in this work.

\section{References}

1. Kumar SK, Dimopoulos MA, Kastritis E, et al. Natural history of relapsed myeloma, refractory to immunomodulatory drugs and proteasome inhibitors: a multicenter IMWG study. Leukemia. 2017;31 (11):2443-2448. doi:10.1038/leu.2017.138

2. Surveillance, Epidemiology, and End Results (SEER) Program. SEER*Stat Database: Populations - Total U.S. (1969-2019), National Cancer Institute, DCCPS, Surveillance Research Program, 2020.

3. Joseph N, Kaufman J, Dhodapkar M, et al. Long-Term follow-up results of lenalidomide, bortezomib, and dexamethasone induction therapy and risk-adapted maintenance approach in newly diagnosed multiple myeloma. $J$ Clin Oncol. 2020;38:JCO.19.02515. doi:10.1200/JCO.19.02515

4. Gandhi UH, Cornell RF, Lakshman A, et al. Outcomes of patients with multiple myeloma refractory to CD38-targeted monoclonal antibody therapy. Leukemia. 2019;33(9):2266-2275. doi:10.1038/ s41375-019-0435-7

5. Carpenter RO, Evbuomwan MO, Pittaluga S, et al. B-cell maturation antigen is a promising target for adoptive T-cell therapy of multiple myeloma. Clin Cancer Res. 2013;19(8):2048-2060. doi:10.1158/ 1078-0432.CCR-12-2422

6. Tai YT, Anderson KC. Targeting B-cell maturation antigen in multiple myeloma. Immunotherapy. 2015;7(11):1187-1199. doi:10.2217/ imt. 15.77

7. Tai YT, Mayes PA, Acharya C, et al. Novel anti-B-cell maturation antigen antibody-drug conjugate (GSK2857916) selectively induces killing of multiple myeloma. Blood. 2014;123(20):3128-3138. doi:10.1182/blood-2013-10-535088

8. Lonial S, Lee HC, Badros A, et al. Belantamab mafodotin for relapsed or refractory multiple myeloma (DREAMM-2): a two-arm, randomised, open-label, phase 2 study. Lancet Oncol. 2020;21 (2):207-221. doi:10.1016/S1470-2045(19)30788-0

9. Trudel S, Lendvai N, Popat R, et al. Antibody-drug conjugate, GSK2857916, in relapsed/refractory multiple myeloma: an update on safety and efficacy from dose expansion phase I study. Blood Cancer J. 2019;9(4):37. doi:10.1038/s41408-019-0196-6

10. Lonial S, Lee C, Badros A, et al. Pivotal DREAMM-2 study: single-agent belantamab mafodotin (GSK2857916) in patients with Relapsed/Refractory Multiple Myeloma (RRMM) refractory to Proteasome Inhibitors (PIs), immunomodulatory agents, and refractory and/or intolerant to anti-CD38 Monoclonal Antibodies (mAbs). Presented at the 25th European Hematology Association (EHA) Congress. Virtual Format: June 11-14; 2020; Poster No EP970; 2020.

11. Lonial S, Lee H, Badros A. DREAMM-2: single-agent belantamab mafodotin (Belamaf) in patients with relapsed/refractory multiple myeloma (RRMM) - 1-year outcomes by prior therapies. Presented at the 2020 ASH Annual Meeting; December 4-8; 2020; Virtual Absract 1417.

12. Cohen AD, Trudel S, Lonial S, et al. DREAMM-2: single-agent belantamab mafodotin (GSK2867916) in patients with Relapsed/ Refractory Multiple Myeloma (RRMM) and High-Risk (HR) cytogenetics. Presented at ASCO 2020 Annual Meeting, June 2020; Poster No 441; 2020.
13. Nooka A, Lee H, Badros A, et al. Infusion-related reaction (IRRs) in the DREAMM-2 study of single-agent belantamab mafodotin (Belamaf) in patients with Relapsed/Refractory Multiple Myeloma (RRMM). Presented at the 2020 ASH Annual Meeting December 48, 2020; Virtual Abstract 3221.

14. Lonial S, Nooka A, Thulasi P, et al. Recovery of ocular events with longer-term follow-up in the DREAMM-2 study of single-agent belantamab mafodotin (Belamaf) in patients with Relapsed or Refractory Multiple Myeloma (RRMM). Presented at the 2020 ASH Annual Meeting December 4-8; 2020; Virtual Abstract 3224; 2020.

15. Eliason L, Correll J, Martin M, et al. Patient-reported experiences during and following treatment with belantamab mafodotin (Belamaf) with Relapsed/Refractory Multiple Myeloma (RRMM) in the DREAMM-2 study. Presented at the 2020 ASH Annual Meeting; December 4-8; Virtual Abstract 3248; 2020.

16. Cohen AD, Lee C, Trudel S, et al. impact of prolonged dose delays on response with belantamab mafodotin (Belamaf; GSK2857916) treatment in DREAMM-2 study: 13 month follow-up. Presented at the Society of Hematologic Oncology (SOHO) Annual Meeting; September 9-12; 2020; Virtual Format; Poster MM-250; 2020.

17. Weisel K, Hopkins T, Fecteau D, et al. Dreamm-3: a phase 3, open-label, randomized study to evaluate the efficacy and safety of belantamab mafodotin (GSK2857916) monotherapy compared with pomalidomide plus low-dose dexamethasone (pom/dex) in participants with Relapsed/Refractory Multiple Myeloma (RRMM). Blood. 2019;134(Supplement_1):1900.

18. Trudel S, McCurdy A, Sutherland H, et al. Part 1 results of a dose finding study of belantamab mafodotin (GSK2857916) in combination with pomalidomide (POM) and Dexamethasone (DEX) for the treatment of relapsed/refractory multple myeloma (RRMM). Presented at the 2020 ASH Annual Meeting; December 4-8; Virtual Abstract 725; 2020.

19. Trudel S, Davis RE, Lewis CE, et al. A phase III study of the efficacy and safety of belantamab mafodotin with pomalidomide and dexamethasone (B-Pd) vs. pomalidomide plus bortezomib and dexamethasone (PVd) in patients with Relapsed/Refractory Multiple Myeloma (RRMM). Presented at the 2020 ASH Annual Meeting; December 48; Virtual Abstract 2302; 2020.

20. Richardson PG, Oriol A, Beksac M, et al. Pomalidomide, bortezomib, and dexamethasone for patients with relapsed or refractory multiple myeloma previously treated with lenalidomide (OPTIMISMM): a randomised, open-label, phase 3 trial. Lancet Oncol. 2019;20(6):781-794. doi:10.1016/S1470-2045(19)30152-4

21. Nooka A, Manteca M, Bahlis N, et al. DREAMM-4: evaluating safety and clinical activity of blantamab mafodotin (belamaf) in combination with pembrolizumab in patients with relapsed/refractory multiple myeloma. Presented at the 25th European Hematology Association (EHA) Congress; Virtual Format; June 11-14, 2020; 2020.

22. Richardson P, Nooka A, Quach H, et al. Dreamm-5 platform trial: belantamb mafodotin (Belamaf) in combination with four differentnovel agents in patients with Relapsed/Refractory Multiple Myeloma (RRMM). Presented at the 2020 ASH Annual Meeting; December 48; Virtual Abstract 2299; 2020.

23. Nooka A, Stockerl-Goldstein K, Quach H, et al. DREAMM-6: safety and tolerability of belantamab mafodotin in combination with bortezomib/dexamethasone in relapsed/refractory multiple myeloma (RRMM). 2020.

24. Popat R, Nooka A, Stockerl-Goldstein K, et al. DREAMM-6: safety, tolerability and clinical activity of belantamab mafodotin (Belamaf) in combination with bortezomib/dexamethasone (BorDex) in Relapsed/ Refractory Multiple Myeloma (RRMM). Presented at the $2020 \mathrm{ASH}$ Annual Meeting; December 4-8; Virtual Abstract 1419; 2020.

25. Rifkin R, Boyd K, Grosicki S, et al. DREAMM-7: a phase III study of the efficacy and safety of belantamab mafodotin (Belamaf) with bortezomib, and dexamethasone (B-Vd) in patients with Relapsed/ Refractory Multiple Myeloma (RRMM). Presented at the 2020 ASH Annual Meeting; December 4-8; Virtual Abstract 3247; 2020. 
26. Cohen AD, Garfall AL, Stadtmauer EA, et al. B cell maturation antigen-specific CAR T cells are clinically active in multiple myeloma. J Clin Invest. 2019;129(6):2210-2221. doi:10.1172/JCI126397

27. Cohen AD, Garfall AL, Dogan A, et al. Serial treatment of relapsed/ refractory multiple myeloma with different BCMA-targeting therapies. Blood Adv. 2019;3(16):2487-2490. doi:10.1182/ bloodadvances.2019000466

28. Maples KT, Joseph NS, Harvey RD. Current developments in the combination therapy of relapsed/refractory multiple myeloma. Expert Rev Anticancer Ther. 2020;20(12):1021-1035. doi:10.1080/147371 40.2020.1828071

29. Schmidt TM, Barwick BG, Joseph N, et al. Gain of Chromosome 1q is associated with early progression in multiple myeloma patients treated with lenalidomide, bortezomib, and dexamethasone. Blood Cancer J. 2019;9(12):94. doi:10.1038/s41408-019-0254-0

30. Tai YT, Landesman Y, Acharya C, et al. CRM1 inhibition induces tumor cell cytotoxicity and impairs osteoclastogenesis in multiple myeloma: molecular mechanisms and therapeutic implications. Leukemia. 2014;28(1):155-165. doi:10.1038/leu.2013.115

31. Tan DS, Bedard PL, Kuruvilla J, Siu LL, Razak AR. Promising SINEs for embargoing nuclear-cytoplasmic export as an anticancer strategy. Cancer Discov. 2014;4(5):527-537. doi:10.1158/2159-8290. CD-13-1005

32. Chari A, Vogl DT, Gavriatopoulou M, et al. Oral selinexor-dexamethasone for triple-class refractory multiple myeloma. $N$ Engl J Med. 2019;381 (8):727-738. doi:10.1056/NEJMoa1903455

33. Suvannasankha A, Kapetanakis V, Prawitz T, et al. MM-209: assessing efficacy via indirect comparison of single-agent belantamab mafodotin (Belamaf; GSK2857916) in DREAMM-2 versus STORM or MAMMOTH studies in Relapsed/Refractory Multiple Myeloma (RRMM). Clin Lymphoma Myeloma Leuk. 2020;20: S300-S301. doi:10.1016/S2152-2650(20)30942-3

34. Chari A, Vogl DT, Jagannath S, et al. Selinexor-based regimens for the treatment of myeloma refractory to chimeric antigen receptor T cell therapy. Br J Haematol. 2020;189(4):e126-e30. doi:10.1111/ bjh. 16550

35. Kashyap T, Argueta C, Aboukameel A, et al. Selinexor, a Selective Inhibitor of Nuclear Export (SINE) compound, acts through NF-kappaB deactivation and combines with proteasome inhibitors to synergistically induce tumor cell death. Oncotarget. 2016;7 (48):78883-78895. doi:10.18632/oncotarget.12428

36. Argueta C, Kashyap T, Klebanov B, et al. Selinexor synergizes with dexamethasone to repress mTORC1 signaling and induce multiple myeloma cell death. Oncotarget. 2018;9(39):25529-25544. doi:10. 18632/oncotarget. 25368

37. Rosebeck S, Alonge MM, Kandarpa M, et al. Synergistic myeloma cell death via novel intracellular activation of caspase-10-dependent apoptosis by carfilzomib and selinexor. Mol Cancer Ther. 2016;15 (1):60-71. doi:10.1158/1535-7163.MCT-15-0488

38. Grosicki S, Simonova M, Spicka I, et al. Once-per-week selinexor, bortezomib, and dexamethasone versus twice-per-week bortezomib and dexamethasone in patients with multiple myeloma (BOSTON): a randomised, open-label, phase 3 trial. Lancet. 2020;396 (10262):1563-1573. doi:10.1016/S0140-6736(20)32292-3

39. Jakubowiak AJ, Jasielec JK, Rosenbaum CA, et al. Phase 1 study of selinexor plus carfilzomib and dexamethasone for the treatment of relapsed/refractory multiple myeloma. $\mathrm{Br} J$ Haematol. 2019;186 (4):549-560. doi:10.1111/bjh.15969

40. Gasparetto C, Schiller G, Callander N, et al. A phase $1 \mathrm{~b} / 2$ study of selinexor, carfilzomib, and dexamethasone (SKd) in Relapsed/ Refractory Multiple Myeloma (RRMM). HemaSphere. 2019;134 (Supplement_1):3157.

41. Gasparetto C, Lentzsch S, Schiller G, et al. Selinexor, daratumumab, and dexamethasone in patients with relapsed/refractory multiple myeloma(MM). J clin oncol. 2020;38(15_suppl):8510. doi:10.1200/ JCO.2020.38.15_supp1.8510
42. Chen A, Bahlis N, Gasparetto C, et al. Selinexor, pomalidomide, and dexamethasone (SPd) in patients with relapsed or refractory multiple myeloma. HemaSphere. 2019;134(Supplement_1):141.

43. Gasparetto C, Lentzsch S, Schiller G, et al. Selinexor, daratumumab, and dexamethasone in patients with relapse/refractory multiple myeloma (MM). J Clin Oncol. 2020;38(15_suppl):8510.

44. White D, Leblanc R, Baljevic M, et al. Seliexor, lenalidomide, and dexamethasone (SRd) for patients with Relapsed/Refractory and Newly Diagnosed Multiple Myeloma. Presented at ASH 2020 Annual Meeting; December 4-7; 2020; Virtual Abstract 1393; 2020.

45. McMillan A, Warcel D, Popat R. Antibody-drug conjugates for multiple myeloma. Expert Opin Biol Ther. 2020;21:1-13. doi:10.1080/14712598.2020.1802422

46. Kumar A, Magdalini M, Bhutani M, et al. Phase 1, first-in-human study of MEDI2228, a BCMA-targeted ADC in patients with Relapsed/Refractory Multiple Myeloma. Presented at the $2020 \mathrm{ASH}$ Annual Meeting; December 4-8; 2020; Virtual Absract 179.

47. Garfall A, Usmani S, Mateos A, et al. Updated phase 1 results of teclistimab, a B-cell Maturation Antigen (BCMA) x CD3 bispecific antibody, in relapsed and/or Refractory Multiple Myeloma (RRMM). Presented at the 2020 ASH Annual Meeting; December 4-8; 2020; Virtual Absract 180.

48. Usmani S, Mateos M, Nahi H. Phase 1 study of teclistimab, a humanized B-cell maturation antigen (BCMA) x CD3 bispecific antibody, in relapsed/refractory multiple myeloma (R/R MM). Presented at the 2020 ASCO Virtual Scientific Program; May 2921; 2020; Abstract 100 .

49. Madduri D, Rosko A, Brayer J, et al. REGN5458, a BCMA x CD3 bispecific monoclonal antibody, induces deep and durable responses in patients with Relapsed/Refractory Multiple Myeloma (RRMM). Presented at the 2020 ASH Annual Meeting; December 4-8; Virtual Absract 291; 2020.

50. Seckinger A, Delgado JA, Moser S, et al. Target expression, generation, preclinical activity, and pharmacokinetics of the BCMA-T cell bispecific antibody EM801 for multiple myeloma treatment. Cancer Cell. 2017;31(3):396-410. doi:10.1016/j.ccell.2017.02.002

51. Costa LJ, Wong S, Bermudez A, et al. First clinical sutdy of the B-cell maturation antigen (BCMA) 2+1 $\mathrm{T}$ cell engager (TCE) CC-93269 in patients (Pts) with Relapsed/Refractory Multiple Myeloma (RRMM): interim results of a phase 1 multicenter trial. Blood. 2019;134(Supp_1):143. doi:10.1182/blood-2019-122895

52. Rodriguez C, D'Souza A, Shah N, et al. Initial results of a phase I study of TNB-383B, a BCMA x CD3 bispecific T-cell redirecting antibody, in relapsed/refractory multiple myeloma. Presented at aSH 2020 Annual Meeting; December 4-7; 2020; Virtual Abstract 293; 2020.

53. Chari A, Berdeja JG, Oriol A, et al. A phase 1, first-in human study of talquetamab, a $\mathrm{G}$ protein-coupled receptor family $\mathrm{C}$ group 5 member D (GPRC5D) x CD3 bispecific antibody, in patients with relaped and/or refractory multiple myeloma (RRMM). Presented at the $2020 \mathrm{ASH}$ Annual Meeting; December 4-8; 2020; Virtual Absract 290.

54. Nakamura R, Lear S, Wilson D, et al. Early pharmacodynamic changes in T-cell activation, proliferation, and cytokine productino confirm the mode of action of BFCR4350A, a FcRH5/CD3 T-cellengaging bispecific antibody, in patients with relapsed/refractory multiple myeloma. Presented at ASH 2020 Annual Meeting; December 4-7; 2020; Abstract 3213; 2020.

55. Cohen $\mathrm{AD}$, Harrison $\mathrm{S}$, Krishnan $\mathrm{A}$, et al. Initial clinical activity and safety of BFCR4350A, a FcRH5/CD3 T-cell-engaging bispecific antibody, in relapsed/refractory multiple myeloma. Presented at the 2020 ASH Annual Meeting; December 4-8; 2020; Virtual Absract 292.

56. Bjorklund CC, Kang J, Lu L, et al. CC-220 is a potent cereblon modulating agent that displays anti-proliferative, pro-apoptotic and immunomodulatory activity on sensitive and resistant multiple myeloma cell lines. Blood. 2016;128(22):1591. doi:10.1182/blood. V128.22.1591.1591 
57. Richardson P, Vangsted A, Ramasamy K, et al. First-in-human phase 1 study of the novel celmod agent CC-92480 combined with dexamethasone in patients with relapsed/refractory myeloma. Presented at EHA 2020, Oral Presentation; Abstract S208; 2020.

58. Raje N, Berdeja J, Lin Y, et al. Anti-BCMA CAR T-cell Therapy bb2121 in relapsed or refractory multiple myeloma. $N$ Engl J Med. 2019;380(18):1726-1737. doi:10.1056/NEJMoa1817226

59. Munshi N, Anderson LD, Shah N, Jagannath S, Berdeja J, Lonial S. Idecabtagene vicleucel (ide-cel;bb2121), a BCMA-targeted CAR T-cell therapy, in patients with relapsed and refractory multiple myeloma (RRMM): initial KarMMA results. J Clin Oncol. 2020;38 (15_suppl):8503. doi:10.1200/JCO.2020.38.15_suppl.8503

60. Alsina M, Shah A, Raje N, et al. Updated results from the phase 1 CRB-402 study of anti-BCMA CAR-T cell therapy bb21217 in patients with relapsed and refractory multiple myeloma: correlation of expansion and duration of response with T-cell phenotypes. Presented at ASH 2020 Annual Meeting; December 4-7; 2020; Abstract 130; 2020.

61. Usmani S, Berdeja J, Truppel-Hartmann A, et al. KarMMa-4: idecabtagene vicleucel (ide-cel), a BCMA-directed CAR T-cell therapy, in high-risk newly diagnosed multiple myeloma. Presented at ASCO 2020 Annual Meeting; June 2020; Abstract 1418; 2020.
62. Madduri D, Berdeja J, Usmani S, et al. CARTITUDE-1: phase 1b/2 study of ciltacabtagene autoleucel, A B-cell maturation antigen-directed chimeric antigen receptor $\mathrm{T}$ cell therapy, in relapsed/refractory multiple myeloma. Presented at ASH 2020 Annual Meeting; December 4-7; 2020; Abstract 177; 2020.

63. Mailankody S, Htut M, Lee C, et al. JCARH125, anti-BCMA CAR T-cell therapy for relapsed/refractory multiple myeloma: initial proof of concept results from a phase $1 / 2$ multicenter study (EVOLVE). Blood. 2018;132:957. doi:10.1182/blood-2018-99113548

64. Cowan A, Pont M, Sather B, et al. Efficacy and safety of fully human BCMA Car-T cells in combination with a gamma secretase inhibitor to increase BCMA surface expression in patients with relapsed or refractory multiple myeloma. Blood. 2019;134(Supplement_1):204. doi:10.1182/blood-2019-129405

65. Rafiq S, Yeku OO, Jackson HJ, et al. Targeted delivery of a PD-1-blocking scFv by CAR-T cells enhances anti-tumor efficacy in vivo. Nat Biotechnol. 2018;36(9):847-856. doi:10.1038/ nbt.4195

\section{Publish your work in this journal}

Clinical Pharmacology: Advances and Applications is an international, peer-reviewed, open access journal publishing original research, reports, reviews and commentaries on all areas of drug experience in humans. The manuscript management system is completely online and includes a very quick and fair peer-review system, which is all easy to use. Visit http://www.dovepress.com/testimonials.php to read real quotes from published authors. 